\title{
Present-self, past-self and the close-other: neural correlates of assigning trait adjectives to oneself and others
}

\author{
Ilona Kotlewska and Anna Nowicka \\ Department of Neurophysiology, Laboratory of Psychophysiology, Nencki Institute of Experimental Biology, 3 Pasteur Street, \\ 02-093 Warsaw, Poland
}

Keywords: event-related potentials, late positive component, positivity bias, self-referential processing, sex-differences

Edited by John Foxe

Received 8 January 2016, revised 25 May 2016, accepted 1 June 2016

\begin{abstract}
Information regarding the past-self may be viewed as information referring to other people. However, evidence supporting this notion at the neural level is rather sparse and it remains unclear whether the past-self is processed like any 'other' or like the close-other only. The aim of this event-related potential study was to investigate this issue. A reflection task requiring evaluation of positive and negative trait adjectives with respect to present- and past-self, a close-other and a famous person was applied. We hypothesized that the past-self and close-other conditions would share their neural underpinnings. The process of reflection on the past-self and close-other was indeed associated with similar mean amplitudes of the late positive component (LPC), whereas in the case of the past-self vs. famous person comparison LPC was significantly enhanced for the past-self. Analogous effects were observed when LPC was calculated for trials with traits judged as either suitable or unsuitable to describe a person who was the target of reflection. Thus, these findings suggest that the processing of information related to the past-self resembles processing of information related to a personally relevant other. Moreover, sex-differences were observed in reaction times and LPC amplitudes for responses reflecting the positivity bias.
\end{abstract}

\section{Introduction}

Questions about the nature of the self have inspired many philosophers and psychologists (James, 1950/1890; Gallagher, 2000). The concept of a single, continuous self has been rejected (Schelling, 1984; Loewenstein, 2000) and now it is widely agreed that the self is not a fixed and completely isolated entity but it is rather a fluctuating, context-dependent, dynamic and complex process (Northoff et al., 2006).

In cognitive and social neuroscience, the topic of the self is addressed by investigations of self-referential processing (SRP). SRP concerns stimuli experienced as strongly related to one's own person, e.g. one's own face, name or body (Lenggenhager et al., 2007; Tacikowski \& Nowicka, 2010). SRP also includes self-mentalizing (Frith $\&$ Frith, 2006) and self-reflection (Moran et al., 2006). Neural underpinnings of SRP have gained much attention with functional magnetic resonance imaging (fMRI) studies showing that SRP is mediated by cortical midline structures (Northoff \& Bermpohl, 2004; Northoff et al., 2006). Event-related potential (ERP) studies, in turn, revealed that SRP is associated mainly with enhanced ERP components: P300 for self-name and self-face (Knyazev, 2013) and late positive component (LPC) for self-reflection (Shestyuk \& Deldin, 2010).

Correspondence: A. Nowicka, as above.

E-mail: a.nowicka@nencki.gov.pl
The self is continually evolving across the life span (Demo, 1992). The impact of temporal perspective on SRP was investigated using faces (Apps et al., 2012; Butler et al., 2013), names (Kotlewska \& Nowicka, 2015) and trait adjectives (D'Argembeau et al., 2008; Luo et al., 2010). Some studies reported differences between neural correlates of the present- and past-self (D'Argembeau et al., 2008), whereas other did not (Luo et al., 2010; Kotlewska \& Nowicka, 2015). In two cases such differences were found when comparing the current self to the self in childhood (Apps et al., 2012; Butler et al., 2013), but they were absent when comparing the current self to the self in adolescence and early adulthood (Butler et al., 2013). Thus this issue needs further investigation.

In addition, the relation between temporally distant selves and others is not fully understood on the neural level. In some studies the past-self was not directly compared to the other (Luo et al., 2010; Apps et al., 2012; Butler et al., 2013). However, two studies reported similar neural underpinnings associated with reflection on the past-self and a friend (D'Argembeau et al., 2008), and with detection of the past (family) self-name and a close-other's name in a group of married women who accepted their husbands' name (Kotlewska \& Nowicka, 2015). Those findings are in line with the notion that people tend to view their past-self as 'another person' (Libby \& Eibach, 2002; Pronin \& Ross, 2006). However, the important issue 
whether it is the case for 'any other' or for a significant-other in particular remains to be resolved. We consider the latter as the valid standpoint and the main assumption of our study, thus hypothesizing similar LPC amplitudes in response to the past-self and the closeother in the trait adjectives reflection task. We were also interested whether LPC findings would reveal any sex-differences as previous fMRI study on self vs. other reflection showed no such effect (Debbané et al., 2014).

\section{Methods}

\section{Participants}

Thirty-two healthy adult participants with normal or corrected-tonormal vision were recruited for the study (16 females). The mean age of participants $( \pm \mathrm{SD}$ ) was $26.3 \pm 1.20$. Two subjects (one female) were excluded from the study due to excessive movement artefacts. None of the subjects reported neural, nor psychological disorders. All the participants were right-handed (controlled with The Edinburgh Inventory: Oldfield, 1971). The study conforms to the World Medical Association Declaration of Helsinki. The experiment was undertaken with the understanding and written consent of each subject, and the experimental protocol was approved by the local Ethics Committee (University of Social Sciences and Humanities, Warsaw, Poland). All subjects received payment for their participation in the study.

\section{Stimuli}

The stimuli consisted of four lists of adjectives representing personal traits. The adjectives were adapted from Anderson's List of Personality-Trait Words in English (Anderson, 1968) and translated into Polish. The frequency of words' occurrence was controlled with the Polish Language Dictionary of Attendance (Kurcz, 1990; Kazojc, 2011).

Four different conditions were used: the self at present (further referred to as present-self), the self in the past (past-self), a closeother person (close-other) and a famous person (famous). Instead of using a pre-defined close-other (e.g. father) we decided - as it was done in our previous research (Tacikowski \& Nowicka, 2010; Tacikowski et al., 2013; Cygan et al., 2014; Kotlewska \& Nowicka, $2015)$ - to ask participants to freely choose their close-other: a person who was the most significant to the participant 'at present', i.e. at the time of our experiment. This was done to avoid a situation in which a pre-defined person (e.g. father) is not really close to a particular subject. Thus, prior to the EEG study participants were requested to assign a closely related person, describe their relationship briefly and report on the length of the relation $(M=10.5$; $\mathrm{SD}=7.9 ; \min =2 ; \max =28)$. Most subjects chose their current partners $(n=23)$, some of them chose a relative $(n=7)$ and only two participants chose their best friends. The length of relationship substantially differed, therefore all experimental data were analysed taking the length of relationship into account.

Four different lists of adjectives were randomly assigned to each condition and rotated across subjects. Each of the four lists contained 40 traits (20 positive and 20 negative) resulting in 160 different words. The lists contents were pseudo-randomized so that no more than three adjectives of the same valence occurred consecutively.

The lists were balanced in respect to word length (i.e. number of letters), frequency of occurrence, and valence and did not differ between each other. A detailed description of the aforementioned parameters may be found in the Supporting Information. The valence (positive/negative) of the words from Anderson's list (Anderson, 1968) was evaluated by a group of five independent judges. Only adjectives with uniform positive or negative judgements were accepted to the study.

\section{Procedure}

Trait adjectives were displayed with white letters against a black background and the size of the stimuli ranged from $2^{\circ} \times 3^{\circ}$ to $2^{\circ} \times 8^{\circ}$. A block design was used to easily separate each condition: present-self, past-self, close-other and famous. To avoid the effect of priming, each word list was split into four sets of 10 items presented within one block and the blocks' order was randomized. The instruction to a particular condition was displayed before the beginning of each block, so 16 times in total.

Participants were instructed to focus their attention on their own person in a particular period of time (present or 5 years ago) or on other people (closely related or famous). Both of the control conditions (close-other and famous) were processed regardless of the time period. The period of 5 years was chosen since it implied that participants had graduated either from their high school or college. At the time of our study the former were college students and the latter were $\mathrm{PhD}$ candidates or had started working. In both cases, there were substantial changes in their lives and the self-concept had to be updated to take these alterations into account (Deutsch et al., 1988; Demo, 1992). Thus the self before graduation from high school or college could be considered as the well-defined past self, whereas the self in the time period following such a 'transition' - as the current/present self.

A detailed instruction was applied prior to each condition to help the participants enter a state of reflection about the particular person, including questions regarding his/her recent or usual behaviour towards the closest people as well as his/her reactions to stressful or difficult situations, and moments of relaxation. The same questions were asked in relation to the period of college or high school to direct attention to their past. Similar questions regarding the closest person were displayed prior to the close-other block. In the famous condition participants were asked to consider how the famous person, i.e. a famous Polish actor/actress (Bogusław Linda for male participants and Krystyna Janda for female participants) might behave on a daily basis, how he/she might react to adversities or act in private relations, etc.

Participants were tasked with judging whether a given adjective was suitable to describe/characterize a person specified in the instruction in a single block (i.e. present-self, past-self, close-other, famous person). Yes/no responses were given by pressing one of two buttons on a Cedrus Response Pad (RB-830, San Pedro, LA, USA). Buttons assignment was counterbalanced on the group level.

The experimental procedure started by displaying the instruction for $9 \mathrm{~s}$. The instruction was followed by a block of 10 words presented in a sequence of events: a blank screen visible for $2 \mathrm{~s}$, a fixation cross visible for $1 \mathrm{~s}$ and a word visible for $7 \mathrm{~s}$ unless the subject responds earlier. A new sequence began upon response or time-out.

\section{EEG recordings}

EEG was continuously recorded from 62 scalp sites using a 128channel amplifier (Quick Amp, Brain Products, Enschede, the Netherlands) and BrainVisionRecorder ${ }^{\circledR}$ software (Brain Products, Gilching, Germany). $\mathrm{Ag}-\mathrm{AgCl}$ electrodes were mounted on an elastic cap (ActiCAP, Munich, Germany) and positioned according to the 
extended 10-20 system. Electrode impedance was kept below $5 \mathrm{k} \Omega$. The EEG signal was recorded against an average of all channels calculated by the amplifier hardware. The sampling rate was $500 \mathrm{~Hz}$.

\section{Behavioural analysis}

Number of yes/no responses and reaction times (RT) were analysed. Only trials with responses within $300-3000$ ms were treated as correct and further analysed. The mean number of segments in each experimental condition was as follows: present-self -39.7 , past-self - 39.8, close-other - 39.6 and famous - 39.7.

Number of yes/no responses was analysed using mixed model repeated measures ANOVA with within-subject factors of 'condition' (at four levels: present-self, past-self, close-other and famous), 'valence' (at two levels: positive vs. negative) and 'type of response' (at two levels: yes vs. no) and between-subject factors of 'sex' (male vs. female) and 'duration of relation with close-other' (short, i.e. less than 8 years vs. long, 8 or more years.). The limit of 8 years was set according to the group median calculated on the basis of the parameter "length of relation with the significant-other' and our group of participants was sub-divided into 'short duration of relation with the close-other' (less than 8 years, 14 subjects, min. 2 years, max. 7 years, $M=4.79$, $\mathrm{SD}=1.76)$ and 'long duration of relationship' ( 8 or more years, 16 subjects, min. 8 years, max. 28 years, $M=15.13, \mathrm{SD}=7.56$ ).

Regarding reaction times, since nine subjects did not respond 'yes' to negative traits either 'no' to positive traits in some conditions, an analysis analogous to that performed for number of yes/no responses (ANOVA with all factors) could not be run. Thus, RTs were first analysed using repeated measures mixed model ANOva with 'condition' (present-self, past-self, close-other and famous) and 'type of response' (yes vs. no) as within subject factors, and 'sex' (male vs. female) and 'duration of relation with the close-other' (short vs. long) as between-subject factors.

This analysis was followed by ANOVA with within-subject factors of 'condition' (present-self, past-self, close-other and famous) and 'positivity bias' (at two levels: positive-yes vs. negative-no) and two between-subjects factors: 'sex' (male vs. female) and 'duration of relation with the close-other' (short vs. long).

All the effects with more than one degree of freedom in the numerator were adjusted for violations of sphericity (Greenhouse \& Geisser, 1959). Bonferroni correction for multiple comparisons was applied to the post hoc analyses. The analyses were conducted in IBM SPSS Statistics 21 Advanced Model.

\section{ERP analysis}

Off-line analysis of EEG data was performed using Brain Vision Analyzer $^{\circledR}$ software (Brain Products $\mathrm{GmbH}$, Munich, Germany). The EEG signal was re-referenced to the mean signal from earlobes. Butterworth Zero Phase filters were applied: low cutoff $-0.1 \mathrm{~Hz}$, $12 \mathrm{~dB} /$ oct; high cutoff $-20 \mathrm{~Hz}, 12 \mathrm{~dB} /$ oct. An additional notch filter $(50 \mathrm{~Hz})$ was applied to remove electrical network artefacts. Independent Component Analysis (ICA; Bell \& Sejnowski, 1995) was used for ocular artefacts correction. After each data set was decomposed into maximally statistically independent components, the elements representing eye blinks were rejected. The remaining components were back-projected to the data after multiplying by the reduced component-mixing matrix. Ocular-artefact-free continuous EEG signal was segmented into epochs extending from $200 \mathrm{~ms}$ before to $1000 \mathrm{~ms}$ after the stimulus onset. Baseline correction was performed with $-200 \mathrm{~ms}$ to $0 \mathrm{~ms}$ interval before the stimulus onset. Artefact rejection was conducted with the following settings: the maximum permitted voltage step per sampling point $-50 \mu \mathrm{V}$; the maximum permitted absolute difference between two values in the segment $-200 \mu \mathrm{V}$; the lowest permitted activity in the $100 \mathrm{~ms}$ interval $-0.5 \mu \mathrm{V}$.

Finally, remaining segments were averaged for each experimental condition to create an ERP for a single subject. Mean amplitudes (i.e. the mean of values at each time point within a certain interval) of the LPC were analysed within the 600-900 ms time-window which was chosen on the basis of previous studies (Luo et al., 2010; Fields \& Kuperberg, 2012).

Selection of electrodes for analyses has to be orthogonal to potential differences between experimental conditions (Kriegeskorte et al., 2009). Thus, such selection has to be done on the basis of topographical distribution of brain activity (in the time window corresponding to a given component), averaged across all experimental conditions. Electrodes within maxima identified in such a topographical map should be further analysed. Based on the topographical distribution of activity in the 600-900 ms time window (see Fig. 2B), collapsed for all experimental conditions (present-self, past-self, close-other, famous), responses (yes, no) and trait valence (positive, negative), electrodes within the region of maximal activity were selected: AFz, AF4, Fz and F2. The data were pooled for those electrodes. This step is justified by the limited spatial resolution of EEG and high correlation between neighbouring electrodes.

Mean LPC amplitudes were analysed using mixed model repeated measures analysis of variance. ANOVA was performed with the withinsubject factor of 'condition' (present-self, past-self, close-other, famous); and between-subject factors of 'sex' (male vs. female) and 'duration of relation' (short vs. long). ERP differences between experimental conditions were further investigated by adding a second within-subject factor of 'type of response' at two levels (yes vs. no). Finally, to take valence into account, ERPs were analysed using repeated measures ANOVA with 'condition' (present-self, past-self, close-other and famous) and 'positivity bias' (positive-yes vs. negative-no) as within-subject factors and with 'sex' (male vs. female) and 'duration of relation' (short vs. long) as between-subject factors.

The latter analysis followed the pattern of behavioural results, which clearly indicated that in the case of positive traits, the vast majority of responses were 'yes', whereas in the case of negative traits the vast majority of responses were 'no'. Thus, trials representing less eligible responses were excluded (i.e. 'yes' for negative and 'no' for positive features) and the remaining segments were used to calculate ERPs for confirmatory responses for positive traits (positive-yes) and denial responses for negative traits (negative-no). The average number of the remaining segments is presented in the Supporting Information. Please note that for some subjects positive/negative traits in some conditions resulted in null yes/no responses and for other subjects, number of such responses was very low (less than 10). For that reason it was not possible to calculate ERPs for positive-no and negative-yes responses.

All effects with more than one degree of freedom in the numerator were adjusted for violations of sphericity (Greenhouse \& Geisser, 1959). Bonferroni correction for multiple comparisons was applied to the post hoc analyses. The analyses were conducted in IBM SPSS Statistics 21 Advanced Model.

\section{Results}

\section{Yes/no responses}

Figure 1A illustrates the mean numbers of yes/no responses for negative and positive traits in each experimental condition. ANOVA 
A
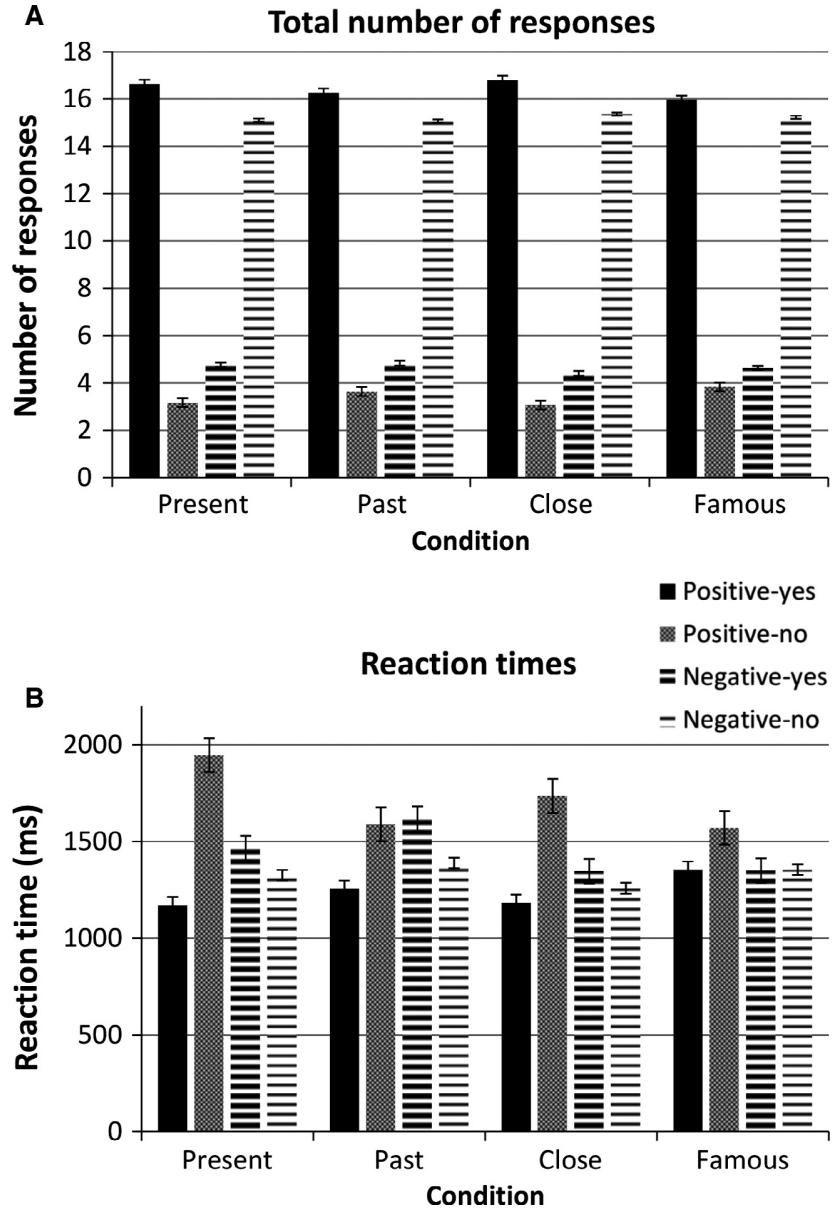

FIG. 1. The number of 'yes' and 'no' responses to positive and negative traits for present-self, past-self, close-other and famous conditions (A). Reaction times split for yes/no responses to positive and negative traits for present-self and past-self, close-other and famous conditions (B).

revealed a significant main factor of 'type of response' $\left(F_{1,26}=16.45 ; P<0.001\right)$. In general, 'yes' responses were chosen more frequently than 'no' responses (see Fig. 1A). The interaction between 'type of response' and 'valence' was also significant $\left(F_{1,26}=339.17 ; P<0.0001\right)$. Post hoc tests indicated that 'yes' responses occurred more often than 'no' responses for positive traits $(P<0.0001)$, whereas for negative words the pattern of responses was reversed and 'yes' responses were less frequent than 'no' responses $(P<0.001)$. All other main effects and their interactions were non-significant.

\section{Reaction times}

Figure 1B illustrates RTs for negative and positive traits in each experimental condition. ANOvA with 'condition' and 'type of response' as within-subject factors and 'sex' and 'duration of relationship' as between-subject factors revealed a significant main factor of 'condition' $\left(F_{3,78}=3.32 ; P=0.024\right)$. The 'condition' $\times$ 'sex' $\left(F_{3,78}=0.95 ; P=0.406\right)$, 'condition' $\times$ 'duration of the relationship' $\left(F_{3,78}=1.07, P=0.360\right)$ and 'condition' $\times$ 'sex' $\times$ 'duration of the relationship' interactions $\left(F_{3,78}=0.42 ; P=0.700\right)$ were not significant. Post hoc comparisons for conditions yielded significant differences between close-other vs. famous $(P=0.048)$; and a tendency between close-other vs. past-self $(P=0.053)$. No other comparisons reached the level of significance: present-self vs. pastself $(P>0.99)$, present-self vs. close-other $(P=0.177)$, present-self vs. famous $(P>0.99)$, past-self vs. famous $(P>0.99)$. In general, the mean RTs for close-other were the shortest among other conditions (mean $\pm \mathrm{SE}$ : present-self $1328.08 \mathrm{~ms} \pm 56.72$; past-self $1358.14 \mathrm{~ms} \pm 76.68$; close-other $1269.87 \mathrm{~ms} \pm 58.41$; famous $1366.75 \mathrm{~ms} \pm 70.21)$.

The same ANOVA design also yielded a significant main factor of 'type of response' $\left(F_{1,26}=12.73 ; P=0.001\right)$. Pairwise comparisons revealed substantially shorter RTs for 'yes' than for 'no' responses (mean \pm SE: $\quad 1300.91 \mathrm{~ms} \pm 65.19$ vs. $1377.96 \mathrm{~ms} \pm 65.41$; $P=0.001)$. Importantly, the interaction of 'condition' $\times$ 'type of response' was significant $\left(F_{3,78}=3.55 ; P=0.025\right)$. Post hoc tests for that interaction revealed significant differences for 'yes' responses, in which the reaction times for close-other were significantly shorter than those for famous person (mean $\pm \mathrm{SE}$ : $1234.30 \mathrm{~ms} \pm 59.66$ vs. $1384.92 \mathrm{~ms} \pm 78.64 ; \quad P=0.002)$. No other pairwise comparisons reached the level of significance.

Subsequently, traits valence and type of response (positive-yes and negative-no responses, referred to as positivity bias) were included into analysis. ANOvA with 'condition' and 'positivity bias' (positive-yes vs. negative-no) as within-subject factors and 'sex' (male vs. female) and 'duration of relation' (short vs. long) as between-subjects factors revealed a significant main factor of 'condition' $\left(F_{3,78}=5.40 ; \quad P=0.004\right)$. Post hoc tests for 'condition' yielded significant differences between close-other vs. past-self $(P=0.021)$ and close-other vs. famous $(P=0.009)$, and a strong tendency between present-self vs. famous $(P=0.051)$. No differences were found between present-self and past-self $(P=0.574)$ or present-self and close-other $(P>0.99)$. These results indicate the shortest RTs for close-other and the longest for the famous condition (see Fig. 1B).

Importantly, 'condition' $\times$ 'positivity bias' interaction was significant $\left(F_{3,78}=3.14 ; P=0.040\right)$. Post hoc comparisons revealed that in the case of positive-yes responses RTs for present-self and for close-other were significantly shorter than those for the famous condition $(P=0.006$ and $P=0.021$ respectively), whereas in the case of negative-no responses RTs for past-self were longer than for close-other $(P=0.013)$. No other pairwise comparisons reached the level of significance: present-self vs. past-self (positive-yes: $P=0.811$; negative-no: $P=0.924)$; present-self vs. close-other (positive-yes: $P>0.99$; negative-no: $P=0.394$ ); present-self vs. famous (negative-no $P>0.99$ ); past-self vs. close-other (positiveyes: $P=0.460$ ); past-self vs. famous (positive-yes: $P=0.775$; negative-no: $P>0.99$ ); close-other vs. famous (negative-no: $P=0.116$ ).

What is more, the significant 'condition' $x$ 'bias' $x$ 'sex' interaction $\left(F_{3,78}=4.83 ; P=0.007\right)$ indicated that findings described above may differ between the two sexes. Post hoc comparisons showed that effects for positive-yes responses were driven by men showing that RTs in the present-self and close-other conditions were substantially shorter than in the famous condition $(P=0.001$ and $P=0.005$ respectively). No other effects reached the level of significance.

All other interactions were insignificant: 'condition' $x$ 'sex' $\left(F_{3,78}=0.99 ; P=0.391\right)$, 'condition' $\times$ 'duration of relationship' $\left(F_{3,78}=0.97 ; P=0.403\right)$, 'condition' $\times$ 'bias' $\times$ 'duration of relationship' $\left(F_{3,78}=0.07 ; P=0.975\right)$ and 'condition' $\times$ 'bias' $\times$ 'duration of relationship' $\times \operatorname{sex}\left(F_{3,78}=1.56 ; P=0.212\right)$.

\section{Electrophysiological results}

Figure 2A presents grand average ERPs in each experimental condition: present-self, past-self, close-other and famous, irrespective of 

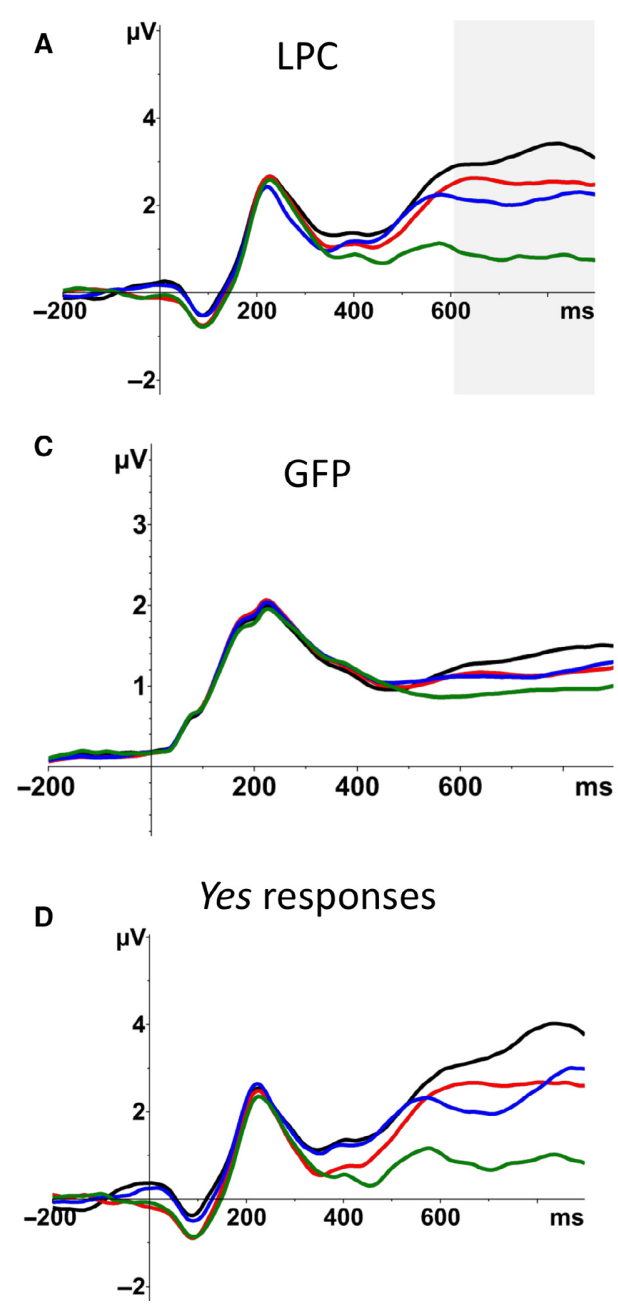

B

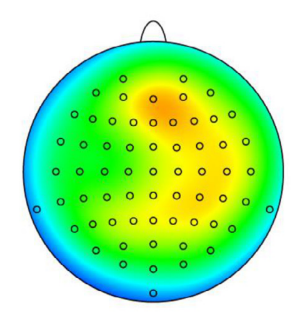

$600 \mathrm{~ms}-900 \mathrm{~ms}$

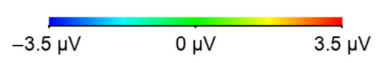

Present self
Past self
Close-other
Famous

No responses

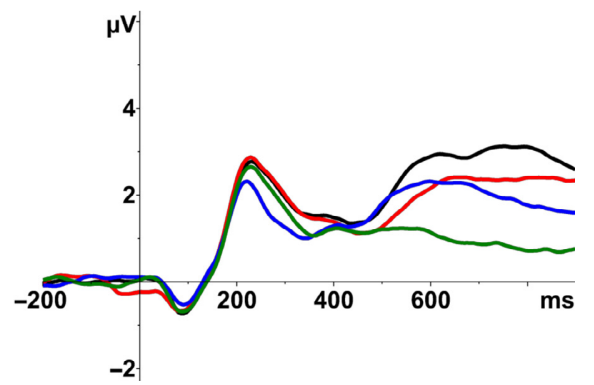

FIG. 2. Grand average ERPs for positive and negative traits taken together, irrespective of participants responses (for pooled $\mathrm{AFz}, \mathrm{AF} 4, \mathrm{Fz}$ and F2) (A). Topographic maps of the voltage distribution in the LPC time window (600-900 ms), collapsed across all experimental conditions (yes/ no responses, negative/positive traits valence and targets of reflection: present-self, past-self, closeother, famous); selection of electrodes to be pooled was based on this topographical distribution of activity (B). Global field power (GFP); GFP quantifies the amount of activity at each time point in the field considering the data from all electrodes simultaneously (C). Grand average ERPs for 'yes' and 'no' responses (D).
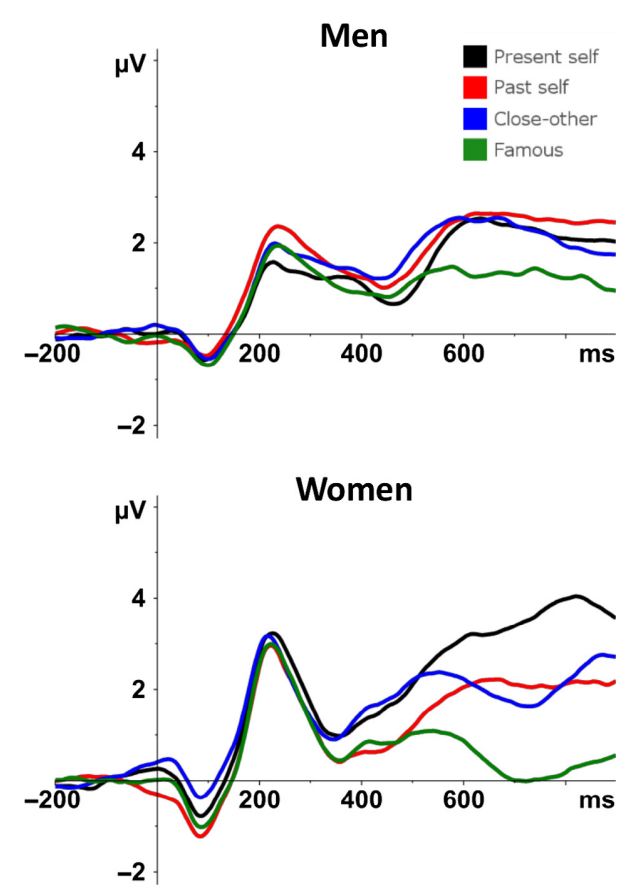

FIG. 3. Grand average ERPs for the positivity bias (positive-yes and negative-no responses) in the group of men and in the group of women. Significant differences between conditions were found in the group of women only. participant responses or trait valence. Such ERPs illustrate changes in brain activity associated with the process of reflection on different targets/persons in general. ANOvA performed on the mean amplitudes of LPC showed the main factor of 'condition' $\left(F_{3,78}=17.27\right.$; $P<0.0001)$. The post hoc tests revealed that mean amplitude of LPC was significantly higher for the present-self than close-other $(P=0.008)$ and famous $(P<0.0001)$ condition. LPC was also significantly enhanced for the past-self and close-other conditions in comparison to famous condition $(P=0.001$ and $P=0.010$ respectively). No differences were found between present-self and past-self $(P=0.320)$, nor past-self and close-other conditions $(P>0.99)$. No interactions were found between the main effect of 'condition' with 'sex' $\left(F_{3,78}=2.09 ; \quad P=0.117\right) \quad$ or 'duration of relation' $\left(F_{3,78}=0.975 ; P=0.409\right)$.

After inclusion of 'type of response' factor into the design, ANOvA yielded again the main effect of 'condition' $\left(F_{3,78}=16.48\right.$; $P<0.0001)$. Interactions of 'condition' with 'sex' $\left(F_{3,78}=1.67\right.$; $P=0.180)$ and with 'duration of relation' $\left(F_{3,78}=1.33 ; P=0.273\right)$ were non-significant. Post hoc tests for the 'condition' factor revealed significant differences between: present-self vs. close-other conditions $(P=0.005)$; present-self vs. famous $(P<0.0001)$, past-self vs. famous $(P=0.001)$ and close-other vs. famous $(P=0.018)$. In all cases, relations between LPC in different conditions mirrored those previously described (i.e. present-self $>$ close-other, present-self $>$ famous, pastself $>$ famous and close-other $>$ famous). No differences were found neither between present-self and past-self $(P=0.142)$, nor between 
past-self and close-other conditions $(P>0.99)$. Figure 2D presents grand average ERPs for 'yes' and 'no' responses in each experimental condition: present-self, past-self, close-other and famous.

ANOVA for mean amplitudes of LPC for positive-yes and negativeno trials showed the significant main effect of 'condition' $\left(F_{3,78}=11.87 ; \quad P<0.0001\right)$ and its interaction with 'sex' $\left(F_{3,78}=3.77 ; P=0.017\right)$. The interaction with 'duration of relationship' was not significant $\left(F_{3,78}=0.94 ; P=0.428\right)$, nor was the three-way interaction 'condition' $x$ 'sex' $x$ 'duration of relationship' $\left(F_{3,78}=0.40 ; P=0.76\right)$.

The post hoc analysis for the 'condition' factor pointed to significantly higher LPC amplitudes for present-self, past-self and closeother in comparison to famous condition $(P<0.0001, P=0.001$ and $P=0.006$ respectively). No differences were found between present-self and past-self $(P=0.968)$, present-self and close-other $(P=0.196)$, or past-self and close-other $(P>0.99)$.

Finally, the post hoc tests for the 'condition' $x$ 'sex' interaction revealed the following effects in women: significantly increased LPC for the present-self in comparison to close-other $(P=0.031)$, and famous $(P<0.0001)$ conditions, as well as for the past-self vs. famous $(P=0.011)$ and close-other vs. famous $(P=0.012)$ conditions. No differences were found between present-self and past-self $(P=0.115)$ or past-self and close-other $(P>0.99)$. However, none of these effects were significant in men: present-self vs. past-self $(P>0.99)$, present-self vs. close-other $(P>0.99)$, present-self vs. famous $(P=0.430)$, past-self vs. close-other $(P>0.99)$, past-self vs. famous $(P=0.105)$ and close-other vs. famous $(P=0.520)$. Thus, these results suggest that in the case of the positivity bias the LPC differences between conditions seem to be driven mainly by women. Figure 3 presents grand average ERPs in men and women for the positivity bias.

\section{Discussion}

A large body of evidence suggests that people distance themselves from their past-self and sometimes regard their past-self as 'another person' (Libby \& Eibach, 2002; Wilson \& Ross, 2003; Pronin \& Ross, 2006). Our study was designed to investigate the electrophysiological correlates of processing information (i.e. evaluation of trait adjectives) referring to the present-self, past-self, close-other and famous person.

In general, our results clearly indicated that the LPC amplitudes associated with the past-self and the close-other conditions were similar. However, LPC amplitudes in the past-self condition in reference to the famous person were significantly enhanced. Thus, these findings suggest that processing information related to the past-self resembles processing information related to a personally relevant other but not just any other. It is worth noting that the length of the relationship with the close-other did not exert a significant influence on the pattern of LPC findings and similar effects of the 'condition' factor were found in both short and long relationship groups.

Similar neural underpinnings of the process of reflection on the past-self and the close-other (friend) were previously reported in one study only (D'Argembeau et al., 2008). In that fMRI study, participants were asked to evaluate their own psychological characteristics and those of an 'other' (friend) for both present and past time periods. The ventral and dorsal medial prefrontal cortex (MPFC) were activated to the same extent by the past-self and close-other condition. Thus, despite different methods of brain activity assessment (fMRI vs. ERP) both studies - D'Argembeau et al. (2008) and the current one - provide converging evidence on similar neural correlates of the past-self and the close-other in the process of reflection on personality traits. It should be noted that processing information other than personality traits resulted in an analogous pattern of findings. In a group of married women who accepted their husband's name, processing the past-self-name (family name) and the closeother's name was associated with equivalent ERP responses (Kotlewska \& Nowicka, 2015). However, it was not the case for past-self and close-other's faces. This may indicate that only information related to non-physical aspects of the past-self were processed similar to the close-other.

Here, similar to our previous studies on SRP (Tacikowski et al., 2011, 2013, 2014; Cygan et al., 2014; Kotlewska \& Nowicka, 2015), the 'close-other' was not pre-defined by the authors (e.g. father), but it was freely chosen by the participants. The vast majority of participants chose their current partners, whereas some of them chose relatives or their best friends. As a consequence, the temporal duration of the relationship varied largely (see the methods section). Thus, one may wonder whether the length of relationship with the close-other exert some impact on the observed lack of LPC differences between the past-self and the close-other. Specifically, one may assume that the longer the relationship the smaller the differences between these two conditions. We investigated this issue thoroughly (see the results section as well as additional analyses in Supporting Information) and there was no effect of the length of acquaintance on observed LPC effects. These results may indicate that it is rather the strength of the emotional bond that matters more than the period of acquaintance per se.

In addition, we observed that the process of reflection on the present-self was associated with enhanced LPC amplitudes in reference to the close-other and famous conditions. This finding is in line with numerous studies on SRP showing the well-known 'self-preference effect' (Gray et al., 2004; Sedikides \& Gregg, 2008; Zhao et al., 2009; Fan et al., 2013; Humphreys et al., 2015). It has been recently proposed that such a self-preference is rather a consequence of enhanced neural activity for self-related stimuli, than an effect of suppression of the other, non-self-related stimulation (Liu et al., 2013). It should be stressed, however, that in the case of positivity bias (i.e. positive-yes and negative-no trials), the difference between LPC amplitudes in the present-self and the close-other conditions was non-significant. This finding may indicate a similarly positive attitude to oneself and the close-other - a person that was chosen by our participants as the most significant in their lives. Self-other integration typically grows from shared experiences, which means that people with whom we have an emotional bond are more likely to be viewed as the self than other people (Gopnik \& Meltzoff, 1994; Slotter \& Gardner, 2009).

Finally, amplitudes of LPC in our study did not differ when the present- and the past-selves were compared. The latter is in line with the findings of Luo et al.'s (2010) study, which reported a lack of differences between late positive ERP responses to the present- and past-selves in the case of psychological traits evaluation. Similarly, differences between ERP responses to present and past self-face as well as to the present and past self-name were non-significant in our (earlier mentioned) study investigating the present and past selves in a group of married women (Kotlewska \& Nowicka, 2015). Moreover, neural correlates of self-face recognition were influenced by the temporal perspective only when the current (adult) self was compared to the 'childhood self' (Apps et al., 2012; Butler et al., 2013). However, such an effect was absent when the current self was compared to the past, but not-so-distant, self (Butler et al., 2013). In contrast, some fMRI studies showed that the MPFC activity was higher when reflecting on the presentself than when reflecting on either the past- or the future-self 
(D'Argembeau et al., 2008, 2010). The issue of similarities/dissimilarities - on the neural level - between the present vs. past selves requires further investigation.

Nevertheless, one may assume that difference in LPC between the present- and past-selves was not detected due to the fact that the past-self was not well defined in this study and the present- and past-selves kind of 'overlap'. If this is the case, similar patterns of ERP responses for the two might be expected, i.e. similar LPC effects for the present-self and the past-self in reference to other conditions (close-other, famous). However, our results pointed out enhanced LPC in the present-self condition in comparison to the 'close-other' and 'famous' conditions in all but the positivity bias analyses, whereas LPC in the 'past-self' condition was increased compared to the 'famous' condition only.

What is more, the analysis of participants' responses revealed a strong bias towards a frequent choice of confirmatory responses to the positive traits and denial responses to the negative traits. Thus, the positivity bias was observed not only to the self, but also to the conditions that regarded other people. Moreover, confirmatory responses for positive traits resulted in significantly shorter RTs for the present-self and the close-other conditions in comparison to the famous person and this effect was mainly driven by men.

Previous studies reported the self-positivity bias at both behavioural and neural levels (Watson et al., 2007). Ma \& Han (2010) conducted a series of behavioural experiments with the use of self-face to distort positive associations with the self. The selfadvantage in face recognition was significantly weaker when the participants' positive view of themselves was questioned. In an ERP study by Watson et al. (2007), participants responded faster to positive self-related trait adjectives than to the negative ones. Also, the positive self-descriptive words resulted in elevated amplitudes at around $500 \mathrm{~ms}$ after the stimulus occurrence. The ERPs were lower for words that did not match the self-positivity bias (Watson et al., 2007; for the correction of figures labels please see: Fields \& Kuperberg, 2012). Frewen et al. (2013), in turn, compared women with positive and negative views of themselves while making judgements about the self and the other. Despite their own attitude, most participants endorsed positive views towards themselves and others, which is congruent with behavioural results reported in this study, i.e. most responses showed positivity bias in all experimental conditions.

In addition, patterns of LPC findings for positive-yes and negative-no trials (the positivity bias) differed in women and men. In the group of women, significant differences were found between conditions (present-self $>$ close-other, present-self $>$ famous, pastself $>$ famous and close-other $>$ famous), whereas they were nonsignificant in the group of men. Sex-differences were not analysed in reference to the self-reflection task in the majority of studies in this field (Fossati et al., 2003; Heatherton et al., 2006; Farb et al., 2007; Watson et al., 2007; D'Argembeau et al., 2008; Esslen et al., 2008; Luo et al., 2010). To the best of our knowledge, only Debbané et al. (2014) used this paradigm to explore the self-reflective processes in adolescent men and women with early expression of psychosis. However, no differences between sexes were found in reflecting about the self and other. In contrast, sex-differences were observed in fMRI study of self-reflection about body image not personal characteristic (Owens et al., 2010). Specifically, participants were instructed to make evaluations of their own bodies in relation to the images displayed. Only female subjects appeared to engage neural mechanisms of self-reflection, as revealed by increased activation in MPFC for overweight vs. thin images. Men showed no significant MPFC activation while processing either type of image. Regarding the sex-differences it is worth to note a kind of dissociation between behavioural and electrophysiological findings in the positivity bias in our study. While in men, the significant differences between conditions were present in RTs and absent in LPC amplitudes, in women such differences were observed in LPC amplitudes and missing in RTs. Thus the issue of sex-differences in the process of self vs. other reflection and in the positivity bias needs further investigation.

Finally, we would like to mention the controversies regarding the topic of trait adjectives lists. There are as many lists as published articles using the self-reflection task in neuroscience. There is no agreement on the lists currently used in research, neither for the number of words nor for the number of lists even within the same language (Alagna \& Hamilton, 1986; Fossati et al., 2003; Heatherton et al., 2006; Farb et al., 2007; Watson et al., 2007; D'Argembeau et al., 2008; Luo et al., 2010). Yet, the spectrum begins from as little as fourteen bipolar trait dimensions of positive and negative affective ratings that result in only 28 words (Alagna \& Hamilton, 1986), and ends up with three lists of ninety separate words summing up to two hundreds and seventy words all together (Heatherton et al., 2006). The discrepancy in the method of trait-adjective evaluation may cause a certain confusion regarding the experimental procedures and reported experimental findings.

In conclusion, processing information (trait adjectives) referring to the past-self and the close-other was associated with similar LPC responses. These results support the notion of similar processing of the past-self and the significant-other, excluding not personally relevant others from the scope of the past-self. Moreover, the LPC to the present-self was enhanced in comparison to other conditions, proving the advantage of self-processing over the processing information about other people in line with numerous SRP studies. Last, but not least, trait adjectives evaluation showed a strong positivity bias towards the self and others.

\section{Conflict of interest}

The authors declare no conflict of interest.

\section{Supporting Information}

Additional supporting information can be found in the online version of this article:

Table S1. Length and frequency of words occurrence in four lists of adjectives used in our study.

Table S2. The number of remained segments (out of 20) after selection of 'yes' responses to the positive traits (positive-yes) and 'no' responses to the negative traits (negative-no).

Appendix S1. Results of an additional LPC analyses for the past-self and close-other conditions.

\section{Acknowledgements}

This work was funded by the National Science Centre, Poland [grant 2012/ 07/B/HS6/01283].

\section{Abbreviations}

ERP, event-related potential; fMRI, functional magnetic resonance imaging; LPC, late positive component; MPFC, medial prefrontal cortex; RT, reaction time; SRP, self-referential processing. 


\section{References}

Alagna, S.W. \& Hamilton, J.A. (1986) Social stimulus perception and selfevaluation effects of menstrual cycle phase. Psychol. Women Quart., 10, 327-338.

Anderson, N.H. (1968) Likableness ratings of 555 personality-trait words. J. Pers. Soc. Psychol., 9, 272-279.

Apps, M.A.J., Tajadura-Jiménez, A., Turley, G. \& Tsakiris, M. (2012) The different faces of one's self: an fMRI study into the recognition of current and past self-facial appearances. Neurolmage, 63, 1720-1729.

Bell, A.J. \& Sejnowski, T.J. (1995) An information-maximization approach to blind separation and blind deconvolution. Neural Comput., 1159, 1129 1159 .

Butler, D.L., Mattingley, J.B., Cunnington, R. \& Suddendorf, T. (2013) Different neural processes accompany self-recognition in photographs across the lifespan: an ERP study using dizygotic twins. PLoS One, 8, e72589.

Cygan, H.B., Tacikowski, P., Ostaszewski, P., Chojnicka, I. \& Nowicka, A. (2014) Neural correlates of own name and own face detection in autism spectrum disorder. PLoS One, 9, e86020.

D’Argembeau, A., Feyers, D., Majerus, S., Collette, F., Van der Linden, M., Maquet, P. \& Salmon, E. (2008) Self-reflection across time: cortical midline structures differentiate between present and past selves. Soc. Cogn. Affect. Neur., 3, 244-252.

D’Argembeau, A., Stawarczyk, D., Majerus, S., Collette, F., Van der Linden, M. \& Salmon, E. (2010) Modulation of medial prefrontal and inferior parietal cortices when thinking about past, present, and future selves. Soc. Neurosci., 5, 187-200.

Debbané, M., Vrti, P., Lazouret, M., Badoud, D., Sander, D. \& Eliez, S. (2014) Self-reflection and positive schizotypy in the adolescent brain. Schizophr. Res., 152, 65-72.

Demo, D.H. (1992) The self-concept over time: research issues and directions. Annu. Rev. Sociol., 18, 303-326.

Deutsch, F.M., Fleming, A., Ruble, D.N. \& Stangor, C. (1988) Informationseeking and maternal self-definition during the transition to motherhood. J. Pers. Soc. Psychol., 59, 420-431.

Esslen, M., Metzler, S., Pascual-Marqui, R. \& Jancke, L. (2008) Pre-reflective and reflective self-reference: a spatiotemporal EEG analysis. NeuroImage, 42, 437-449.

Fan, W., Chen, J., Wang, X., Cai, R., Tan, Q., Chen, Y., Yang, Q., Zhang, S. et al. (2013) Electrophysiological correlation of the degree of self- reference effect. PLoS One, 8, e80289.

Farb, N.A., Segal, Z.V., Mayberg, H., Bean, J., Mckeon, D., Fatima, Z. \& Anderson, A.K. (2007) Attending to the present: mindfulness meditation reveals distinct neural modes of self-reference. Soc. Cogn. Affect. Neur., 2 313-322.

Fields, E.C. \& Kuperberg, G.R. (2012) It's all about you: an ERP study of emotion and self-relevance in discourse. NeuroImage, 62, 562-574.

Fossati, P., Hevenor, S.J., Graham, S.J., Grady, C., Keightley, M.L., Craik, F. \& Mayberg, H. (2003) In search of the emotional self: an FMRI study using positive and negative emotional words. Am. J. Psychiatry, 160, 1938-1945.

Frewen, P.A., Lundberg, E., Brimson-Théberge, M. \& Théberge, J. (2013) Neuroimaging self-esteem: a fMRI study of individual differences in women. Soc. Cogn. Affect. Neur., 8, 546-555.

Frith, C.D. \& Frith, U. (2006) The neural basis of mentalizing. Neuron, 50, 531-534.

Gallagher, S. (2000) Philosophical conceptions of the self: implications for cognitive science. Trends Cogn. Sci., 4, 14-21.

Gopnik, A. \& Meltzoff, A.N. (1994) Minds, bodies, and persons: young children's understanding of the self and others as reflected in imitation and theory of mind research. In Parker, S.T., Mitchell, R.W. \& Boccia, M.L. (Eds), Self-Awareness in Animals and Humans: Developmental Perspectives. Cambridge University Press, Cambridge, pp. 166-186.

Gray, H.M., Ambady, N., Lowenthal, W.T. \& Deldin, P. (2004) P300 as an index of attention to self-relevant stimuli. J. Exp. Soc. Psychol., 40, 216-224.

Greenhouse, S. \& Geisser, S. (1959) On methods in the analysis of profile data. Psychometrika, 24, 95-112.

Heatherton, T.F., Wyland, C.L., Macrae, C.N., Demos, K.E., Denny, B.T. \& Kelley, W.M. (2006) Medial prefrontal activity differentiates self from close others. Soc. Cogn. Affect. Neur., 1, 18-25.

Humphreys, G.W., Sui, J., Humphreys, G.W. \& Sui, J. (2015) Attentional control and the self: the Self-Attention Network (SAN). Cogn. Neurosci., 8928.
James, W. (1950) The Principles of Psychology (vol 1). Dover Publications (original work published in 1890), New York.

Kazojc, J. (2011) Slownik frekwencyjny jezyka polskiego V.05.2011.

Knyazev, G.G. (2013) EEG correlates of self-referential processing. Front Hum. Neurosci., 7, 1-14.

Kotlewska, I. \& Nowicka, A. (2015) Present self, past self and close-other: event-related potential study of face and name detection. Biol. Psychol., 110, 201-211.

Kriegeskorte, N., Simmons, W.K., Bellgowan, P.S.F. \& Baker, C.I. (2009) Circular analysis in systems neuroscience - the dangers of double dipping. Nat. Neurosci., 12, 535-540.

Kurcz, I. (1990) Słownik Frekwencyjny Polszczyzny Wspótczesnej. Polska Akademia Nauk, Instytut Jezyka Polskiego, Kraków.

Lenggenhager, B., Tadi, T., Metzinger, T. \& Blanke, O. (2007) Video ergo sum: manipulating bodily self-consciousness. Science, 317, 1096-1099.

Libby, L.K. \& Eibach, R.P. (2002) Looking back in time: self-concept change affects visual perspective in autobiographical memory. J. Pers. Soc. Psychol., 82, 167-179.

Liu, Y., Sheng, F., Woodcock, K.A. \& Han, S. (2013) Oxytocin effects on neural correlates of self-referential processing. Biol. Psychol., 94, 380-387.

Loewenstein, G. (2000) Emotions in economic theory and economic behavior. Am. Econ. Rev., 90, 426-432.

Luo, Y., Huang, X., Chen, Y., Jackson, T. \& Wei, D. (2010) Negativity bias of the self across time: an event-related potentials study. Neurosci. Lett., 475, 69-73.

Ma, Y. \& Han, S. (2010) Why we respond faster to the self than to others? An implicit positive association theory of self-advantage during implicit face recognition. J. Exp. Psychol. Hum. Percept. Perform., 36, 619-633.

Moran, J.M., Macrae, C.N., Heatherton, T.F., Wyland, C.L. \& Kelley, W.M. (2006) Neuroanatomical evidence for distinct cognitive and affective components of self. J. Cogn. Neurosci., 18, 1586-1594.

Northoff, G. \& Bermpohl, F. (2004) Cortical midline structures and the self. Trends Cogn. Sci., 8, 102-107.

Northoff, G., Heinzel, A., de Greck, M., Bermpohl, F., Dobrowolny, H. \& Panksepp, J. (2006) Self-referential processing in our brain - A meta-analysis of imaging studies on the self. NeuroImage, 31, 440-457.

Oldfield, R.C. (1971) The assessment and analysis of handedness: the Edinburgh inventory. Neuropsychologia, 9, 97-113.

Owens, T.E., Allen, M.D. \& Spangler, D.L. (2010) An fMRI study of selfreflection about body image: sex differences. Pers. Indiv. Differ., 48, 849 854.

Pronin, E. \& Ross, L. (2006) Temporal differences in trait self-ascription: when the self is seen as an other. J. Pers. Soc. Psychol., 90, 197-209.

Schelling, T.C. (1984) The intimate contest for self-command. In Schelling, T.C. (Ed.), Choice and Consequence. Harvard University Press, Cambridge, MA, pp. 57-82.

Sedikides, C. \& Gregg, A.P. (2008) Self-enhancement food for thought. Perspect. Psychol. Sci., 3, 102-116.

Shestyuk, A.Y. \& Deldin, P.J. (2010) Automatic and strategic representation of the self in major depression: trait and state abnormalities. Am. J. Psychiatry, 167, 536-544

Slotter, E.B. \& Gardner, W.L. (2009) Where do you end and i begin? Evidence for anticipatory, motivated self - other integration between relationship partners. J. Pers. Soc. Psychol., 96, 1137-1151.

Tacikowski, P. \& Nowicka, A. (2010) Allocation of attention to self-name and self-face: an ERP study. Biol. Psychol., 84, 318-324.

Tacikowski, P., Brechmann, A., Marchewka, A., Jednoróg, K., Dobrowolny, M. \& Nowicka, A. (2011) Is it about the self or the significance? An fMRI study of self-name recognition. Soc. Neurosci., 6, 98-107.

Tacikowski, P., Brechmann, A. \& Nowicka, A. (2013) Cross-modal pattern of brain activations associated with the processing of self- and significant other's name. Hum. Brain Mapp., 34, 2069-2077.

Tacikowski, P., Cygan, H.B. \& Nowicka, A. (2014) Neural correlates of own and close-other's name recognition: ERP evidence. Front. Hum. Neurosci., 8, 194

Watson, L.A., Dritschel, B., Obonsawin, M.C. \& Jentzsch, I. (2007) Seeing yourself in a positive light: brain correlates of the self-positivity bias. Brain Res., 1152, 106-110.

Wilson, A.E. \& Ross, M. (2003) The identity function of autobiographical memory: time is on our side. Memory, 11, 137-149.

Zhao, K., Yuan, J., Zhong, Y., Peng, Y. \& Chen, J. (2009) Event-related potential correlates of the collective self-relevant effect. Neurosci. Lett., 464, 57-61. 